\title{
Volume of Distribution Steady State Predicted by Bound Drug
}

National Cancer Institute

\section{Source}

National Cancer Institute. Volume of Distribution Steady State Predicted by Bound Drug. NCl Thesaurus. Code C156575.

The volume of distribution at steady state based on the predicted CLST for a substance administered by extravascular dosing, divided by the fraction of bound drug. 